EPJ Web of Conferences 32, 03008 (2012)

DOI: $10.1051 /$ epjconf/20123203008

(C) Owned by the authors, published by EDP Sciences, 2012

\title{
ECE AND ECH APPLICATION FOR INVESTIGATION OF PLASMA SELF-ORGANIZATION AT T-10 TOKAMAK
}

\author{
V.I. Poznyak, T.V. Gridina, V.V. Pitersky, G.N. Ploskirev, E.G. Ploskirev, O. Valencia \\ RRC “Kurchatov Institute”, IFT, 123182, Kurchtov Sq. 1, Moscow, Russia, pozn@tokamak.ru
}

\begin{abstract}
A behaviour of the high energy electrons appointed by their ECE simultaneously on two first harmonics are used for the electron distribution function analysis. Experiments were fulfilled in Ohmic regime and with on-axis ECH. The explored spectrum of emission from the extreme column periphery comes into existence after a relaxation of the primary flow of electrons with energy more than $1 \mathrm{MeV}$ on the plasma waves. Maximum energy of the generated electrons does not vary during all discharge time also as the spectrum shape of its emission. The form of spectrum does not depend on electron temperature and density but its character width is directly proportional to the value of magnetic field. The appointed connection of the dynamical features between the peripheral high energy electrons and the periodical kinetic instability in the central plasma area (mode $\mathrm{m} / \mathrm{n}=1 / 1$ ) confirms an existence of the wave transport from the center to the plasma edge. The set of experimental data corresponds to the theory of the stationary electron distribution function formation by the potential plasma waves which apparently are the main mechanism of plasma self-organization in tokamak.
\end{abstract}

\section{Introduction}

The plasma self-organization is put into effect only by the accidentally emergent wave processes which possess by a fast far-ranging transport. The best mechanism of the plasma selforganization is the potential electron plasma waves. They are capable to carry an electron momentum to a long distance but do not leave the plasma volume. Those waves exist in any plasma, in any conditions. Their velocities are maximal among all kind of the potential waves. The wave transport of the electron momentum leads to an alteration of the holding magnetic field structure. The connection between micro and macro plasma parameters appears. A spontaneous wave generation is more active under an influence of the internal and external forces. This process into frames of the probability theory is described by the electron distribution function change.

The goal of this work is to investigate the regularity of electron distribution function dynamics and its connection with the internal self-optimizing structural peculiarities of the plasma system. Main means of the analysis in this work is electron cyclotron emission of the high energy electrons. Fig. 1 shows the full spectra into the ranges of $1^{\text {st }}$ and $2^{\text {nd }}$ ECE harmonics. Similar spectra of emission comprising the slow changing background component and series of the short-time $(10-30 \mu \mathrm{s})$ spikes of a high amplitude emission are observed in all discharge types (magnetic field $\mathrm{B}_{\mathrm{t}}=23-26 \mathrm{kGs}$ ) under the condition that plasma density in its external boundary (LFS) does not exceed the critical value for the ordinary waves with frequencies 39-59 GHz. The high frequency parts of both spectra $(60-77$ and 119-175 GHz) are the thermal plasma emission on $1^{\text {st }}$ and $2^{\text {nd }}$ resonance correspondingly. It was determine that the low frequency parts of both spectra are ECE of the high energy electrons [1]. The measuring emission arises from the narrow in vertical direction $(\sim 5 \mathrm{~cm})$ and along radius $(\sim 2 \mathrm{~cm})$ plasma area on LFS. The first harmonic emission was identified as ECE in ordinary polarization. Such 
waves propagate only between the plasma cut-off line and chamber wall practically without absorption. This is confirmed by that that ECE spectrum does not depend on positions of antenna (LFS or HFS) and polarizer in antenna output.
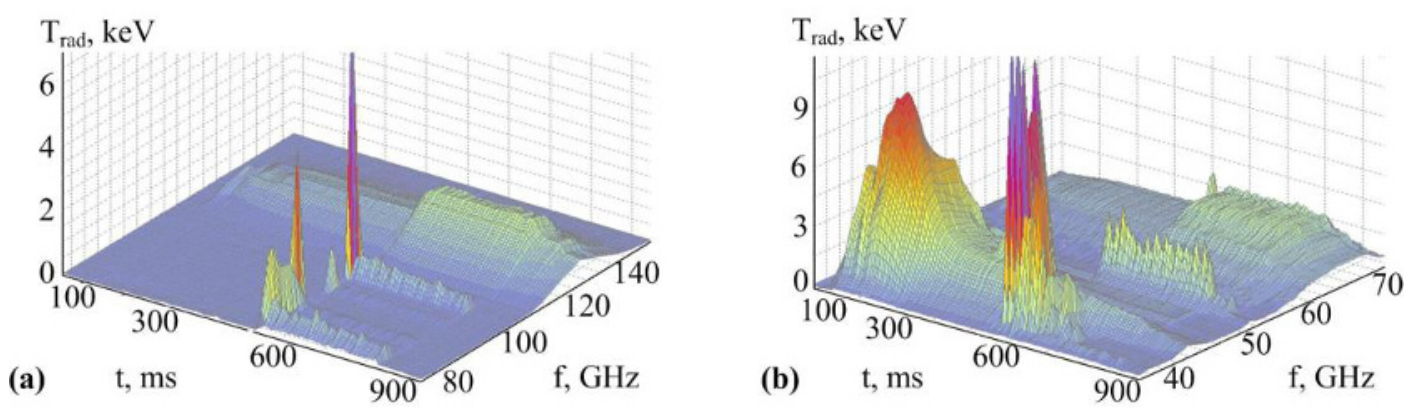

Figure. 1. \#36055. 3D-shape of full ECE spectra. (a) - Second harmonic; (b) - first harmonic.

\section{Main discharge characteristics}

We use on-axis ECH (140 GHz, power up to $1 \mathrm{MW}$ ) for a striking demonstration of the plasma self-organization phenomena. Off-axis ECH $(129 \mathrm{GHz}$, up to $0.8 \mathrm{MW})$ is applied in addition. The discharge parameters are: magnetic field $\mathrm{B}_{\mathrm{t}}(0)=25 \mathrm{kGs}$, average density $\mathrm{n}_{\mathrm{e}}=(1.5-2) 10^{13} \mathrm{~cm}^{-3}$, plasma current $I_{p}=250 \mathrm{kA}$. In such conditions the emission zone is situated in the vicinity of the magnetic surface $\mathrm{q}=3$. ECH leads to essential amplification of the global plasma oscillations in mode $\mathrm{m} / \mathrm{n}=1 / 1$ up to $50 \%$ of a slow changeable level (fig. 2a) [2].

(a)
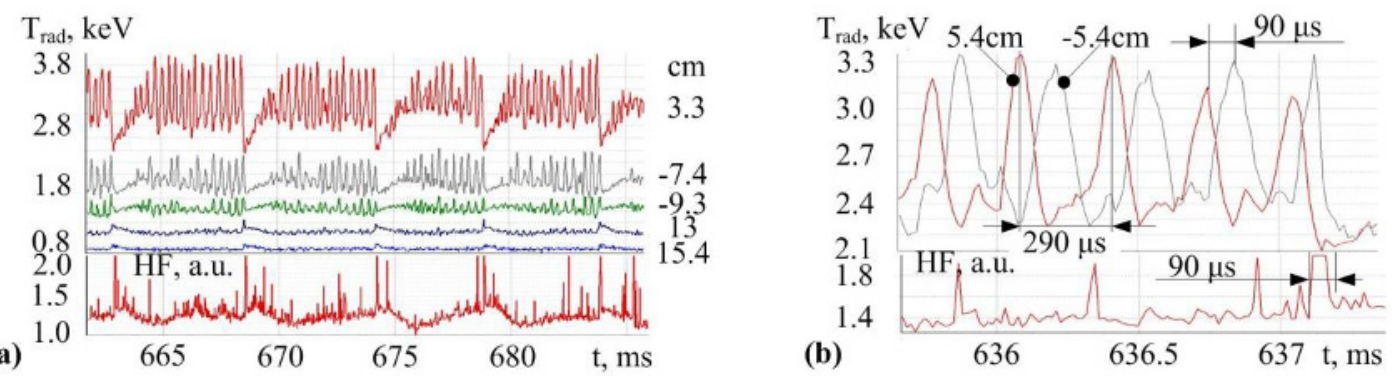

Figure 2. \# 36057. ECE and plasma noise HF in the regime with saturation of oscillating part of signals (three on-axis gyrotrons). (a) - Top - ECE signals in direct and reverse sawtooth phase, bottom - variation of low changing part and spikes of monitor HF signals (integral of plasma oscillations into frequency range $0.5-16$ $\mathrm{GHz}$ ) in instability acts. (b) - Top - detection of eigen oscillations period, here $t_{\text {eigen }} \sim 90 \mu \mathrm{s}$; bottom - signal HF. High amplitudes of HF on both figures are limited by comparator.

The amplitude disturbances of the signals in second ECE harmonic move along magnetic force lines unevenly. The measuring velocities of the disturbance passage from LFS to HFS determine the frequencies of circulation around the torus. The transit time $90 \mu \mathrm{s}$ on fig. $2 \mathrm{~b}$ is equal here to the period of frequency $\sim 11 \mathrm{kHz}$. Those frequencies are in good correspondence with the frequencies of current oscillations which are measured by magnetic probes [3]. Notably they are the characteristics of the eigen plasma oscillations. Their values $\mathrm{f}^{1 / 1} \sim 6.2-6.5 \mathrm{kHz}, \mathrm{f}^{2}{ }_{1 / 1}$ $\sim 10-12 \mathrm{kHz}$ и $\mathrm{f}_{1 / 1}^{4} \sim 22-24 \mathrm{kHz}$ are practically permanent during all discharge. Upper index stands a number of harmonic, lower - mode structure.

The space structure of oscillations under their strong build-up is being more complicate during on-axis $\mathrm{ECH}$. New rational surface $\mathrm{q}^{*}=1$ springs up on radius $\mathrm{r}=4.5 \mathrm{~cm}$ that is equal to 
half of the main surface $q=1$ radius $r=8.7 \mathrm{~cm}$ [4]. A motion of disturbances inside surface $\mathrm{q}^{*}$ $=1$ quickens defining the doubled frequency relative to frequency determined by driving surface $\mathrm{q}=1$ in the regime of the weak oscillation. A motion of the disturbances out the surface $\mathrm{q}^{*}=1$ on part of their trajectory from HFS to LFS occurs twice slowly than in case of weak oscillations. This effect determines frequency of oscillating process repetition $\mathrm{f}^{1}$ mod $\sim 3.6 \mathrm{kHz}$.
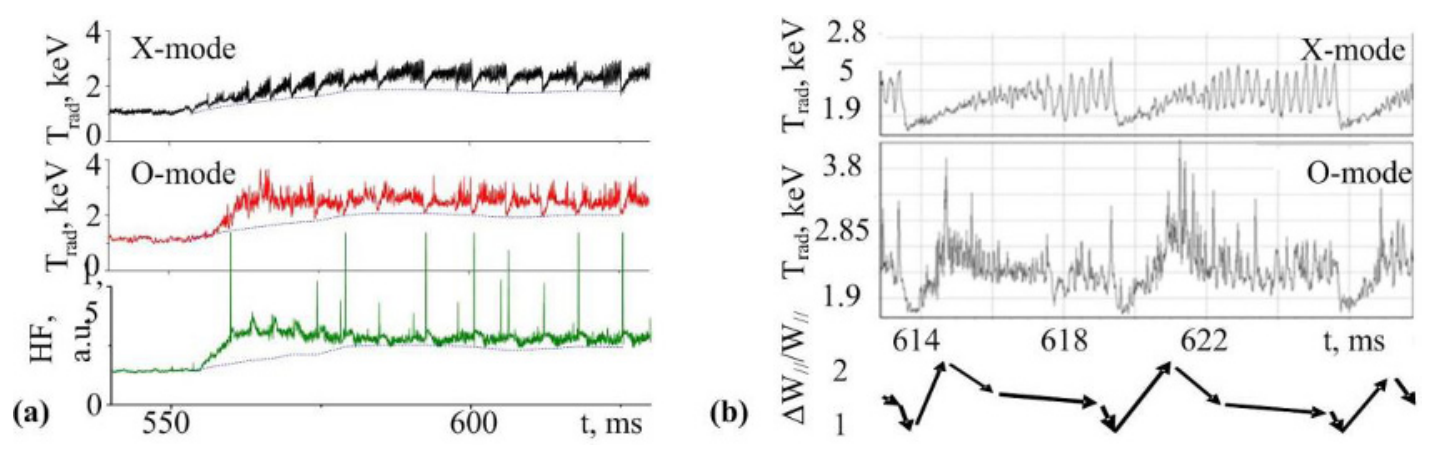

Figure 3. (a) - Dynamics of X-ECE, O-ECE and plasma noise HF in the first state of ЕCH. (b) - Х- и О-ЕСЕ in quasi-stationary stage. Graph below illustrates a variation of longitudinal energy relative to its minimal value just after internal disruption during sawtooth cycle.

It was discovered by simultaneous ECE measurements in two polarizations from plasma with high optical depth that energy in main part of distribution pumps over from longitudinal degree of freedom to transverse degree: fast in every period of global oscillations and relatively slow in every period of sawtooth process. It means that the periodical kinetic instability takes place [2]. Strong oscillations both relaxation and background parts of O-ECE signals are observed under high ECH power (fig. 3). Variations of the longitudinal velocity can be comparable with its average value pending period of "saw". An equality of O-ECE and X-ECE signals just after every internal disruption shows to the establishment of the almost equilibrium distribution function. Maximal deformation of distribution function happens in the first phase of $\mathrm{ECH}$. The diagram of the plasma noise signal (HF) in this time is similar to that of O-ECE that corresponds to the driving role of the electron plasma waves in a creation of certain shape of electron distribution function.

\section{ECE spectra of peripheral high energy electrons}

Fig. 4a shows the diagnostics data in the initial state of discharge: loop voltage, plasma current, density, electron temperature, spectral lines, O-ECE signals, HF monitor and other. Any signs of the discharge breakdown are absent during first $4 \mathrm{~ms}$ after the switching-on of the vortex field $(100 \mathrm{~ms})$. Only some increase of the molecular (Cont) and atomic (Dalfa) lines testifies a weak exposure of electric field to the main plasma. Electrons in the high electric field $(\sim 30 \mathrm{mV} / \mathrm{cm})$ reach to energy $\sim 1 \mathrm{MeV}$ per $1 \mathrm{~ms}$ passing fast the energy region $\sim 100 \mathrm{eV}$ when they are able to ionized effectively the deuterium atoms. Their velocities approach to the speed of light and creating them current is saturated. In the sequel energy of field expends only to the increase of mass. However the considerable level of HF signal (here is limited by comparator) is more two orders of value higher than that in quasi-stationary stage. This indicates the strong plasma wave excitation. Really the spectral components $2-8 \mathrm{GHz}$ (fig $4 \mathrm{~b}$ ) can represent the plasma wave dynamics. In the same time it is obvious that frequencies $10-24 \mathrm{GHz}$ are higher than electron plasma frequency during first $4 \mathrm{~ms}$. It means that those frequencies can be only ECE of electrons with energy considerably more than $1 \mathrm{MeV}$. Any emission in this range disappears when the powerful relaxation process stops. As result secondary flow of electrons with energy no more than $200 \mathrm{keV}$ is generated as the O-ECE signals show. Distribution function of this electrons remains all discharge time and executes only short-time oscillations 
relative certain position. The dynamics of the second flow O-ECE corresponds clear to the plasma density growth. In this time the avalanche ionization happens and the rotation transformation forms in the chamber center.
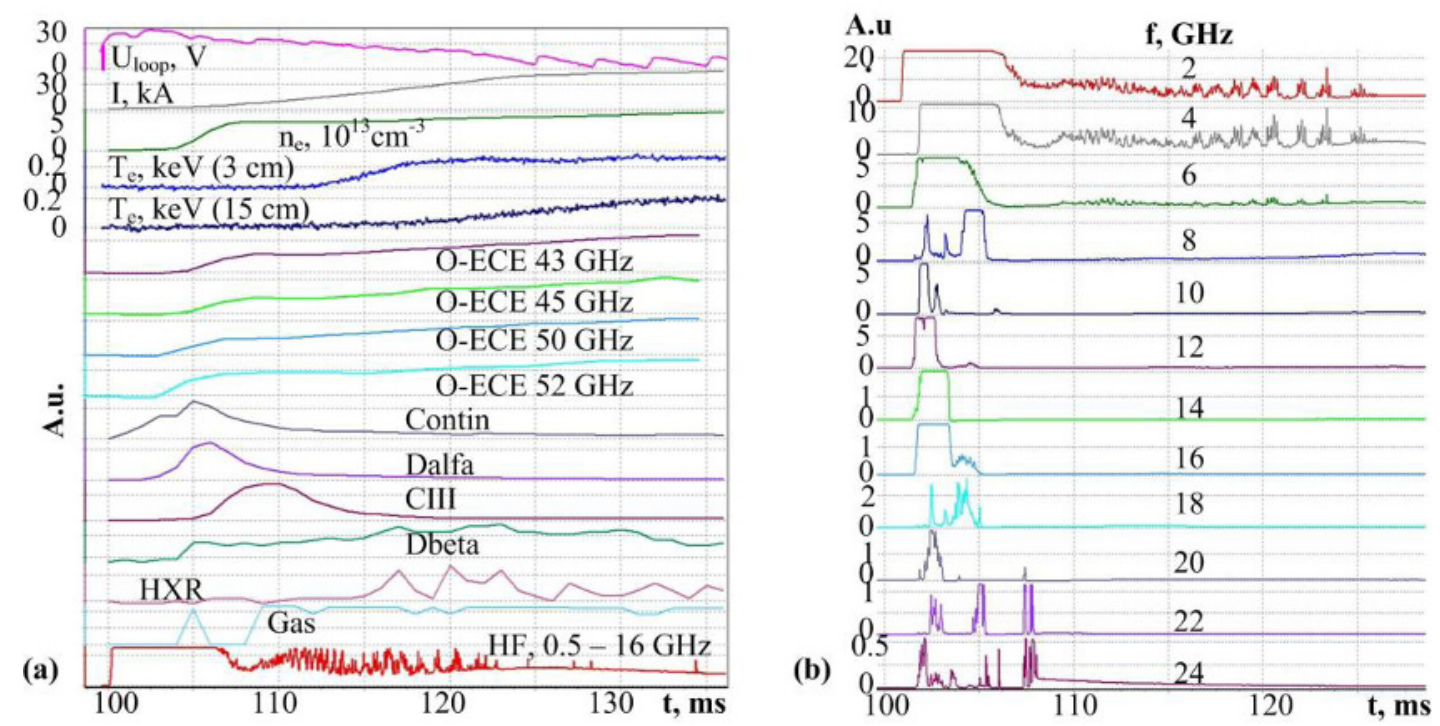

Pис. 4. \#36058. Initial stage of discharge. (a) - Main plasma parameters. (b) - Spectral components of electromagnetic emission.

Fig. 5 illustrates a dynamic of the background part of the first resonance $\mathrm{O}$-mode spectrum. In the first stage after breakdown, ECE intensity increases without any changes of a spectrum form (fig. 5a). Amount of the high energy electrons grows with the conservation of their distribution function. The beginning of spectrum relaxation precedes an appearance of sawtooth oscillations in the central plasma zones (5b). The emission power decreases, the spectral maximum shifts to the low frequencies that is energy of electrons diminishes. Such scenario lasts $\sim 100 \mathrm{~ms}$. Then the spectrum goes back to the primary form. Without $\mathrm{ECH}$, the spectrum maintains its shape up to the end of discharge. The switching-on of the on-axis ECH distorts spectrum to the low frequencies (fig. 5c, curve 1) during the electron temperature grows phase. It means that a fall of Coulomb friction intensifies the accelerative process. Then the spectrum is reinstated anew (curves 2-6). The central ECH is accompanied by new peculiarity 55-57 GHz. Its maximum accomplishes the strong pulsations with a frequency of the sawtooth repetition. In this pulse the weak peculiarity arises in Ohmic stage. Fig. 5e shows spectrum after one of three central gyrotron switching off. Fig. $5 \mathrm{f}$ - only one off-axis gyrotron operates.

Fig. 6 shows the changes of the second ECE harmonic spectrum in different discharge phases. The level of ECE emission in the low frequency part of spectrum in Ohmic phase is many times lower than in thermal frequency range. Only small ascending grade is observed near the low frequency boundary. Under the powerful central ECH switching-on, ECE intensity in this range is being comparable with the maximal level of emission in the thermal range. Similar effect corresponds to the growth of the perpendicular electron energy near the energy spectrum boundary owing to the turn of electron velocity under interactions with waves on the abnormal Doppler resonance. The peculiarity on frequencies $110-115 \mathrm{GHz}$, which are multiple to the frequencies on first resonance, appears in addition. This phenomenon was discovered also on others installations, particularly ASDEX [5]. A reduction of on-axis ECH power decreases the amplitude of this maximum up to its disappearance as in this pulse (fig 6b, curves 1-2). Off-axis ECH does not create this effect. 

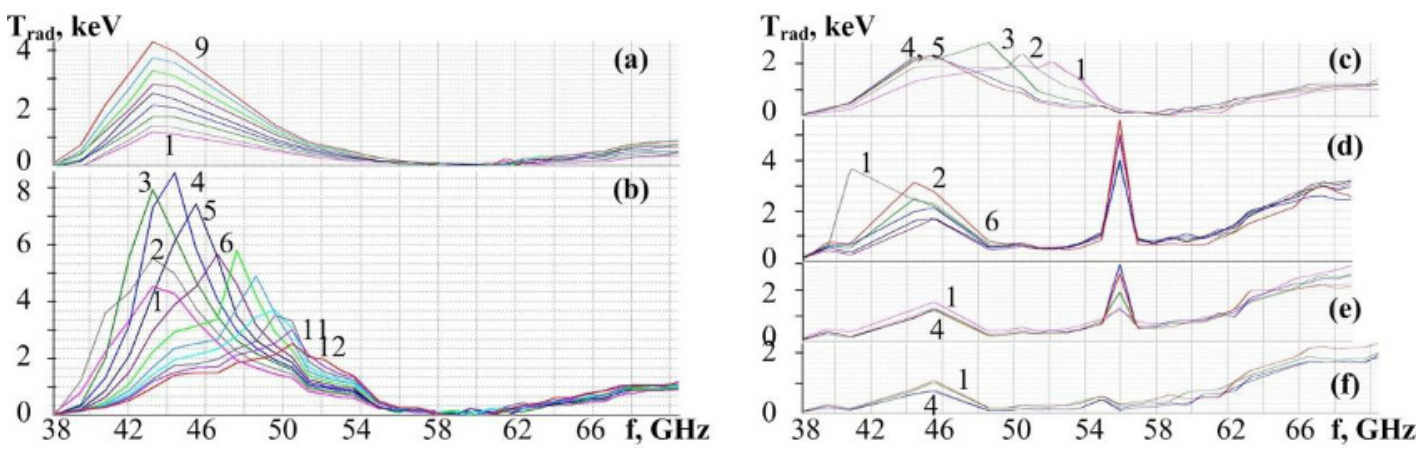

Figure 5. \#36057. Dynamics of the first harmonic ECE spectrum. (a) - The consistency of spectrum shape in the current ramp up stage (110-200 ms, the curves were obtained through interval $10 \mathrm{~ms})$; (b) - spectrum relaxation (200-310 ms, $10 \mathrm{~ms})$; (c) - restoration of spectrum form (310-550 ms, $60 \mathrm{mc})$; (d) - after switchingon of three on-axis and one off-axis gyrotrons $(560-680 \mathrm{~ms}, 20 \mathrm{Mc})$; (e) - spectrum shape after one on-axis gyrotron switching-off (690-790 ms $20 \mathrm{mc})$; (g) - one off-axis gyrotron (800-880 ms, $20 \mathrm{~ms})$.
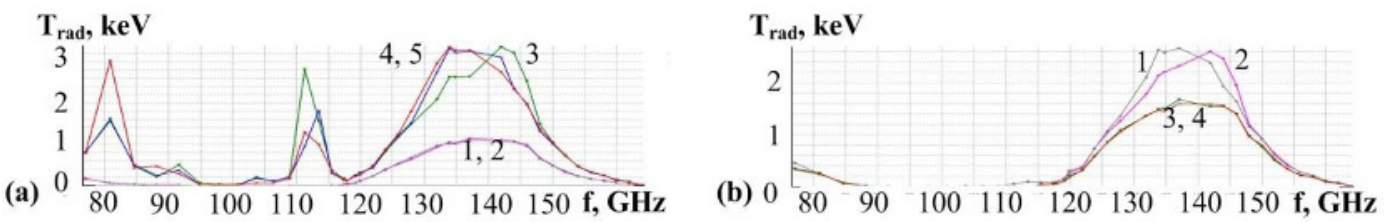

Fig. 6. \#36057. Second harmonic ECE spectrum. (a) - 1 and 2 - Ohmic stage; $3-5$ - three on-axis gyrotrons and one off-axis. (b) - 1 and 2 - two on-axis and one off-axis gyrotrons; 3 and 4 - only off-axis gyrotron.

A generation of spikes happens also by certain lows. The first spikes appear in the phase of a spectrum restoration (fig. 5c) only after internal disruptions. The current frequencies decrease (fig. 7a) that corresponds to the growth of energy in a relaxation range. ECE amplitudes increase considerably with the ECH start. They can fill almost all period of "saw". On the second ECE harmonic, splashes of the high amplitude happen only just after ECH start.

Fig. $7 \mathrm{~b}$ represents a full dynamics of $\mathrm{O}-\mathrm{ECE}$ spectrum background during discharge. In the first phase, spectrum preserves its form, than the frequency of maximum reduces during $\sim 50 \mathrm{~ms}$ and returns to the initial position after $\sim 100 \mathrm{~ms}$. A reduction of Coulomb friction after ECH switch-on leads to the short-time intensification of the accelerating process (a decrease of spectrum frequencies). Similar effect is observed in this time in main part of distribution in the central plasma area (fig. 3a). The spectral peculiarity $55-56 \mathrm{GHz}$ does not change also. It was discovered earlier that rational magnetic surfaces possess the property to accumulate electrons with the high longitudinal energy [6]. The position of the zone emitting main maximum of O-mode ECE is in good correlation with the position of surface $\mathrm{q}=3(28 \mathrm{~cm})$. We assume that emission with frequencies $55-$ $56 \mathrm{GHz}$ is provided by electrons which are on the surface $\mathrm{q}=2$. A great number of factors show to that that the peripheral electron population is a consequence of the wave transport from the central area. Those are the different correlations with sawtooth process, equality of frequencies both splashes appearance and global oscillations in mode $\mathrm{m} / \mathrm{n}=1 / 1$ and others. O-ECE signals contain also the modulating component with a frequency of sawtooth repetition. Oscillations into the ranges 5052 and $53-55 \mathrm{GHz}$ have the opposite phase that shows to a swing of electron energy spectrum. The longitudinal energy oscillations in central area have just that character (fig. 3b). Taking into account integration with time constant 25-30 ms (life-time of the high energy electrons) several times more than saw period, we will observe simultaneously two smoothed spectral maxima. Consequently this spectral dynamics is similar to the splitting of spectral line that the dotted arrow illustrates on fig $7 \mathrm{~b}$. It is important to emphasize that the spectrum form does not depend on electron temperature and 
density. The width of spectrum (from the cyclotron frequency on the plasma boundary to the frequency of maximum) is in direct proportion to the value of magnetic field.
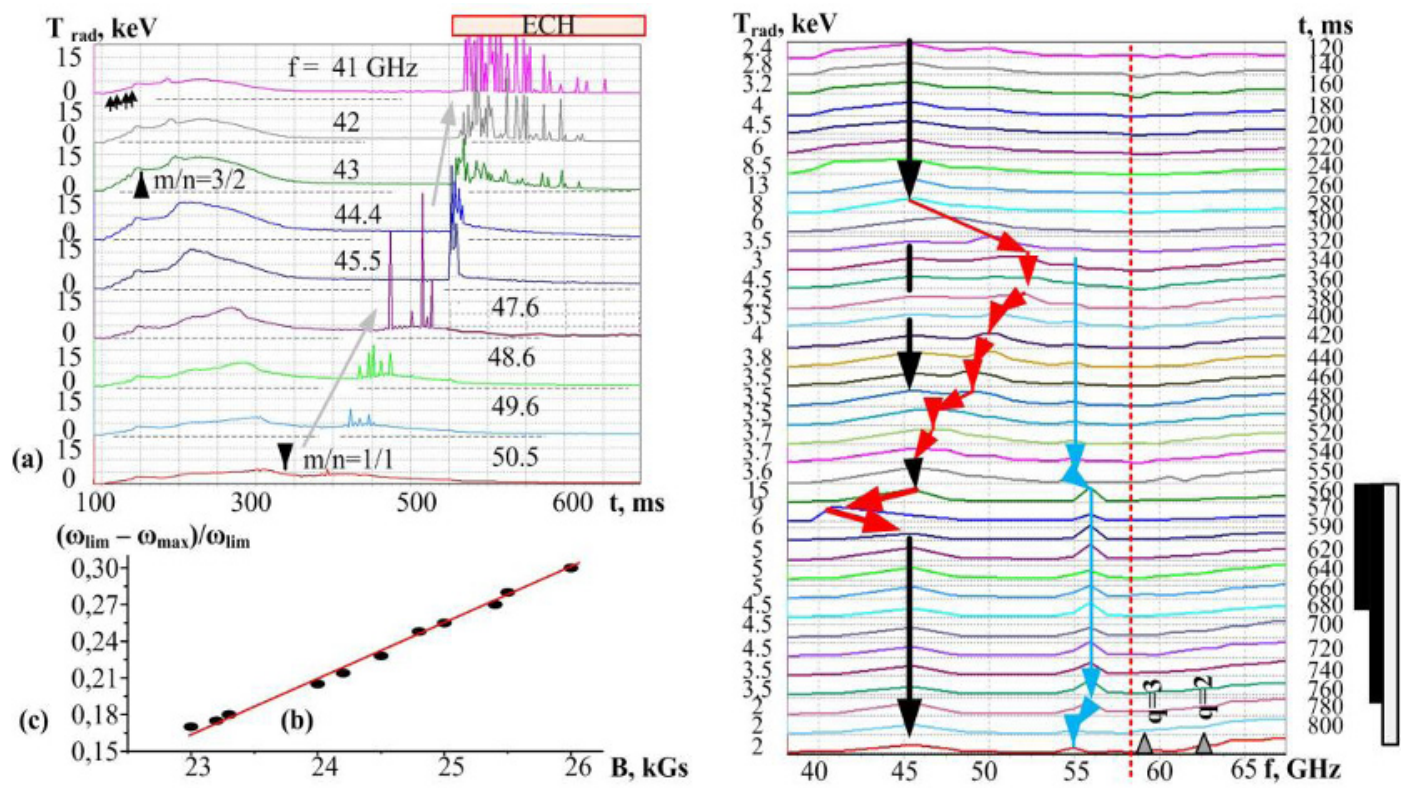

Fig. 7. Peculiarities of O-ECE spectrum. (a) - \#36055. O-ECE signals. The oblique arrows show reduction in time of the current frequencies whereon a spikes generation are registered. The vertical arrows fixed excitation times for different modes of the global plasma oscillation. (b) - \#36057. Dynamics of O-ECE spectrum background. Radiation temperature in spectral maximum in different time $\mathbf{t}$ is denoted in the left ordinate. The dotted line is plasma boundary by rail limiter. Arrows show a change of spectral maxima pozition, $\Delta$ is coordinates of rational magnetic surfaces. Operation time of on-axis gyrotrons is showed by black color, offaxis gyrotron - by white color. (c) - dependence of spectrum width on magnetic field value.

\section{Summary}

In plasma with electric field, the stationary electron distribution function is established by the action of electron plasma waves as it was shown in a whole number of theoretical works, for example [7, 8]. A plateau with boundary conditions $\mathrm{v}_{\mathrm{c}}<\mathrm{v}_{/ /}<\mathrm{v}_{\mathrm{D}}$ (look at fig. 8) and small longitudinal velocity $\left(\mathrm{v}_{/ /}\right)$derivative is formed if $\omega_{\mathrm{pe}} / \omega_{\mathrm{ce}}<1\left(\omega_{\mathrm{pe}}\right.$ and $\omega_{\mathrm{ce}}$ correspondingly electron plasma and cyclotron frequencies). Oscillations exciting on Cherenkov resonance and spreading in small angles relative to magnetic field direction play the constitutive role here. $\mathrm{v}_{\mathrm{c}}$ is the critical velocity when the electric force is equal to the Coulomb friction force. In the area $v_{/ /}>v_{D}$, waves exciting on the abnormal Doppler resonance are driving force. Upper boundary of plateau $v_{D}$ does not depend on electron temperature and density but has the functional dependence on magnetic and longitudinal electric field $\mathrm{v}_{\mathrm{D}} \sim \mathrm{B} / \sqrt{\mathrm{E}}$ Fig. 8 illustrates a change of distribution function by a growth both temperature and density (the current ramp up phase) in the constant operating electric field. It can confront the change of maximum position in O-ECE spectrum that is proportional to magnetic field with the introduced dependence for $\mathrm{v}_{\mathrm{D}}$ taking into account that ECE power connects linearly with the longitudinal energy. We think that the current pinch velocity increases in the last stage of the current penetration to the central plasma area enhancing the electric field and shortening the electron distribution tail (reduction of $v_{D}$ value) that ECE measurements show as relaxation. 
(a)

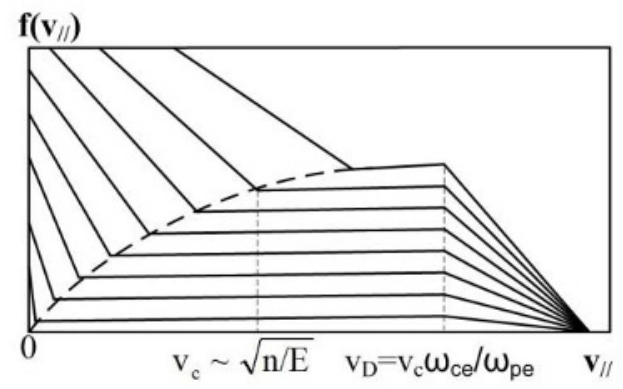

Fig. 8. Schematic illustration of distribution function change with temperature and density grows after breakdown for constant operating electric field $\mathrm{E}$. (b)

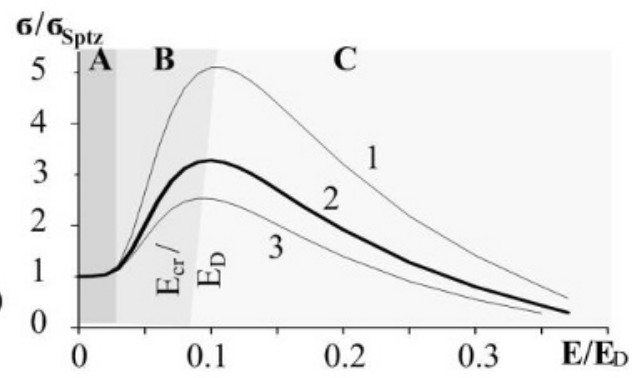

Fig. 9. Dependence of plasma electric conductivity on electric field for different relations $\omega_{\mathrm{pe}} / \omega_{\mathrm{ce}}: 1-0.5,2-0.7,3-0.9$.

If we know a shape of distribution function we can obtain the dependence of plasma electric conductivity on electric field (fig. 9) [9]. It can separate out three character areas in this dependence. In area $\mathbf{A}$ with $\mathrm{E}<\mathrm{E}_{\text {run }}$, the conductivity corresponds to the Spitzer formula. The flow of high energy electrons is negligible and their life-time is enough small. Therefore their emission cannot be measured by ECE diagnostics. Area B corresponds to so-called runway regime. The superthermal emission is registered the easier than value $\omega_{\text {pe }} / \omega_{\text {ce }}$ lower. In area $\mathbf{C}$ with $\mathrm{E}>\mathrm{E}_{\mathrm{cr}} \sim 0.1 \mathrm{E}_{\mathrm{D}}$, current and electric field are connected by the positive feedback that is the reason of the observing kinetic instability in central plasma region when the fast irreversible compression of electron distribution and correspondingly current decay happen. It should to understand that realization of instability is possible only in uniform plasma which we have in experiment.

(a)

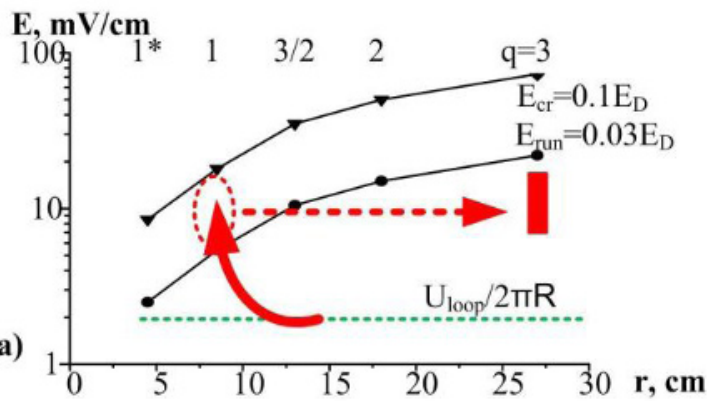

(b)

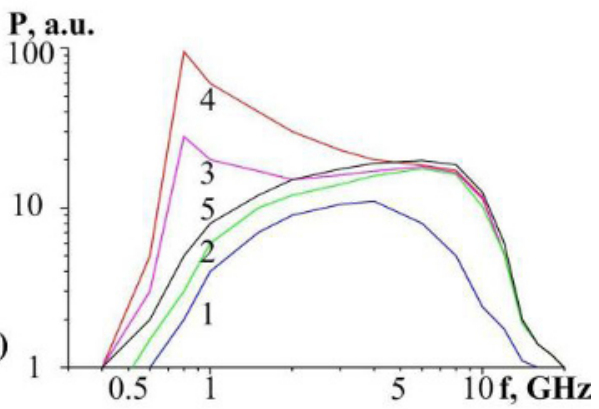

Fig. 10. - \#36057. Creation of high energy periphery electron population.
Fig. 11. \#36058. Plasma waves spectral dynamics. $1-\mathrm{OH}$ stage. Later - with on-axis ECH. 2 - in stable stage of sawtooth: 3 - before. 4 - iust in and 5 - after disruption.

Fig. 8 shows an interval of the electric field values (with taking into account of the real temperature and density distributions) when considerable amount of electrons can be accelerated to high energies. The rectangle on the graph captures the range of the necessary fields (in vertical direction) in the space area (in horizontal direction). It is obvious that the direct acceleration of electrons at the plasma edge by electric field cannot provide sufficient level of ECE. But such process is possible into central plasma zone under the condition that real electric field is 2-6 time higher than value $U_{\text {loop }} / 2 \pi R$ (as the curved arrow shows). Thus an appearance of the high energy electrons at the edge of plasma column is a result of wave transport from the plasma center (dotted arrow). The formation of the second maximum in ECE spectra is apparently conditioned be similar processes into zone inside the surface $q^{*}=1$ which appears under a strong build-up of the global oscillations in $\mathrm{m} / \mathrm{n}=1 / 1$ mode.

Extraction of distribution function of the high energy electrons in the plasma periphery [10] shows that they are strongly trapped (and ripple trapped). Their perpendicular energy is ten times more at the average than the longitudinal one. This is in compliance with measured spectrum of 
plasma oscillations (fig. 11). Plasma oscillation spectrum has the bell like form which is provided mainly by $\mathrm{q}=2$ wave excitation. Such spectrum collapses fully under disruption according to $\mathrm{q}=2$ surface. The alteration of plasma state during sawtooth process is accompanied by a change of plasma oscillation spectrum only in the narrow frequency band less than 7-8 GHz (curves 3 and 4 , above bell like spectrum by $\mathrm{q}=2$ ). Upper boundary of $1^{\text {st }}$ high frequency oscillation mode (by $\mathrm{q}=1$ ) is $\sim 42 \mathrm{GHz}$ (according density on radius $\mathrm{q}=1$, see [11]). Only waves with low frequencies can reach the plasma edge. They propagation angles can be evaluated according to expression $\omega \sim \omega_{\text {pe }} \cos \Theta$, where $\Theta$ is angle between magnetic field direction and wave vector $\mathbf{k}$. As far as for potential waves $\mathbf{k} \| \mathbf{v}$, only almost transverse waves provided by interactions of trapped electrons inside $\mathrm{q}=1$ surface can reproduce them at the plasma edge. Oblique waves cannot reach peripheral area by plasma cut-off and absorb along plasma radius against density profile. A growth of energy storage inside of $\mathrm{q}=2$ surface after internal disruption leads to the strengthening of wave excitation by $\mathrm{q}=2$ surface (boundary frequency $\sim 18 \mathrm{GHz}$ ). This secondary process can determine the growth of high frequency part of the measured spectrum (higher $8 \mathrm{GHz}$ ) and can provide more weak trapped electrons.

Hereby experiments on $\mathrm{T}-10$ show that spectrum of the peripheral electrons preserves its shape during all discharge. Only short-time deviations of frequency: upwards (reduction of electron energy) with completion of current profile establishment and downwards (growth of electrons energy) with the fast growth of electron temperature after start of on-axis ECH - happen. The spectrum form does not depend on electron temperature and density but its width is in direct proportion to magnetic field. Great number of dynamic characteristics of spectra shows to that the appearance of the high energy electrons on the plasma periphery becomes formed by the wave transport from the central plasma area. The consistency of the measured spectrum and its dependence on magnetic field shows that the reason of the stationary shape of spectrum is the potential plasma waves exciting on abnormal Doppler resonance. The observing during all discharge kinetic instability (mode $\mathrm{m} / \mathrm{n}=1 / 1$ including internal disruptions) as we assume is the consequence of the periodical nonlinear current pinch creating the critical value of electric field.

This work was supported by the Russian Ministry of Education and Science (Contract No. 16.518.11.7004) and Rosatom (Contract No. H.4f.45.90.11.1021).

1. V.I. Poznyak, V.V. Piterskii, G.N. Ploskirev, E.G. Ploskirev, Proc. of $17^{\text {th }}$ Joint Workshop on ECE and ECH, Deurne, The Netherlands (2012)

2. V.I. Poznyak, T.V. Gridina, V.V. Piterskii, at al., Plasma Physics Report, 8 (2012) (to be published)

3. V.I. Poznyak, T.V. Gridina, V.V. Piterskii, at al., Proc. of $16^{\text {th }}$ Joint Workshop on ECE and ECH, Sania, China, 114 (2010)

4. V.I. Poznyak, A.M. Kakurin, T.V. Gridina at al., Plasma Physics Report, 9 (2012) (to be published)

5. A.G. Peeters and W. Suttpor, Proc. of $10^{\text {th }}$ Joint Workshop on ECE and ECH, Ameland, The Netherlands, 403 (2008)

6. V.I. Poznyak, V.V. Piterskii, G.N. Ploskirev, E.G. Ploskirev, Proc. of $15^{\text {th }}$ Joint Workshop on ECE and ECH, Yosemite National Park, California, USA, 256 (2008)

7. K. Papadopulos, B. Hui, N. Winsor, Nuclear Fusion, 17, 1087 (1977)

8. Mushcietti L., Appert K., Vaclavic J., Phys. Fluids, 25, 1187 (1982)

9. Poznyak V.I., Bagdasarov A.A., Piterskii V.V. et al., Proc. of $15^{\text {th }}$ Int. Conf. on Plasma Phys. and Control. Nucl. Fusion Research, Seville, Spain, 1994, Nucl. Fusion, 2, 169 (1995)

10. P.V. Minashin, A.B. Kukushkin, V.I. Poanyak, Proc. of $17^{\text {th }}$ Joint Workshop on ECE and ECH, Deurne, The Netherlands (2012)

11. Poznyak V.I., Piterskii V.V. at al., Proc. of $17^{\text {th }}$ Joint Workshop on ECE and ECH, Deurne, Netherlands, (2012) 